\title{
Assessment of morphological damages of leaves of selected plant species due to vehicular air pollution, Kolkata, India
}

\author{
Abantika Nandy, Soumendra Nath Talapatra*, Pritha Bhattacharjee, \\ Punarbasu Chaudhuri, Aniruddha Mukhopadhyay \\ Department of Environmental Science, University of Calcutta \\ 51/1 \& 2 Hazra Road, Kolkata 700019, India \\ *Phone: 91-33-2461-5445 \\ *E-mail address: ecologylive@yahoo.co.in
}

\begin{abstract}
The present study deals to know morphological damages of leaves of four selected plant species near roadside due to vehicular air pollution in Kolkata, India. The selected plant species are Ficus bengalensis, Ficus religiosa, Alstonia scholaris and Neolamarckia cadamba as these are very common as avenue trees. The study area was selected as per Low vehicular load (LVL) as control area, moderate vehicular load (MVL) area, high vehicular load (HVL) area and heavy vehicular load (HeVL) These three sampling stations were selected on the basis of moderate, high and heavy traffic density and continuous vehicular movement as per visualization. The control area was considered as time dependent vehicular movement due to less traffic density. The morphological damages with special reference to length (L), breadth (B) and L/B ratio and visible injuries in leaves. The visible injuries such as pigmentation, chlorosis, necrosis and burning of leaves of four selected species is documented. There was an increasing and decreasing tends in all four plant species at all three vehicular emission exposed sites (MVL, HVL and HeVL) compared to control site (LVL). In all experimental sites such as MVL, HVL and $\mathrm{HeVL}$, the extra growth and reduction pattern significantly ( $\mathrm{P}<0.001,0.01$ or 0.05$)$ observed when compared to control site (LVL) for L, B and $\mathrm{L} / \mathrm{B}$ ratio. The visible injuries (in \%) of leaves were also observed in increasing trends. This study is a preliminary assessment of tolerant species that already have been used in greenbelt development to protect air pollutants as well as biological monitoring to know exact load of automobile air pollution but further researches are needed in relation to biochemical and genetic damage study. It was observed that out of four selected species Ficus bengalensis, Alstonia scholaris and Neolamarckia cadamba are more tolerant species and Ficus religiosa is a less tolerant species because of these may have fighting abilities by waxy coatings, accumulation and degradation abilities to vehicular air pollution at all exposed area when compared to control area.
\end{abstract}

Keywords: Air pollution; Vehicular pollution; Leaf Injuries; Biomonitoring; Foilar morphology

\section{INTRODUCTION}

Air pollution causes by industrial activities, domestic combustions, automobiles etc. Air pollution by automobiles is a matter of great concern in India and physico-chemical analysis 
of vehicular air pollutants revealed that still Kolkata is more susceptible to air pollution (CPCB, 2009; Citizen's Report, 2011). The expansion of city causes may be many fold increase in the number of automobiles.

Ministry of Environment and Forest (MoEF), Government of India has prescribed $33 \%$ plantation under greenbelt development plan for any developmental activities such as industries, urban development etc. The Kolkata city is decorated by roadside plantation by very common plant species. The concept of greenbelt development is mainly to protect air pollution from industries, automobiles etc. The plant species under greenbelt can effectively used as air pollutants prevention as resistant (accumulator or tolerant) and sensitive (Warren, 1973; Singh and Rao, 1983; Tiwari and Tiwari, 2006). The plant species are suitable to know an alarming indication of air pollution by showing mainly foliar damages more in sensitive species and/or less in tolerant species.

Many studies as bioindicator plants showing visible leaf injuries, morphological, anatomical anomalies and biochemical changes related to air pollution have been studied by many countries of the globe (Middleton et al., 1956; Bull and Mansfield, 1974; Husen et al., 1999; Naveed et al., 2010; Seyyed and Koochak 2011) as well as other parts of India (Tiwary et al., 2008; Saquib et al., 2010; Deepalakshmi, 2013). The studies have already been done on physico-chemical analysis by air pollution in Kolkata but no one has attempted easy screening of bioindicator plant species near roadside as biomonitoring for heavy vehicular loads and their air pollutants exposure.

The present study aims to know morphological damages of leaves of plant species near roadside due to vehicular air pollution in Kolkata, India.

\section{MATERIALS AND METHODS}

Kolkata with a population of 4.5 million as of 2011, the $3 \mathrm{rd}$ largest metropolitan area in India, centrally positioned at Latitude $=22^{\circ} 34^{\prime} \mathrm{N}$, Longitude $=88^{\circ} 21^{\prime} \mathrm{E}$ and covers an area of $1,026 \mathrm{~km}^{2}$. Situated at 5.18 meters above sea level.

The study areas were selected as per vehicular loads. The study was carried out at 3 sampling stations viz (i) Low vehicular load (LVL) as control area at Sonarpur station road, (latitude $=22^{\circ} 26^{\prime} \mathrm{N}$ and longitude $=88^{\circ} 25^{\prime} \mathrm{E}$ ) (ii) moderate vehicular load (MVL) area as Hazra Road (latitude $=22^{\circ} 31^{\prime} \mathrm{N}$ and longitude $=82^{\circ} 21^{\prime} \mathrm{E}$ ), (iii) high vehicular load $(\mathrm{HVL})$ area as Asutosh Mukherjee Road near Exide point (latitude $=22^{\circ} 32^{\prime} \mathrm{N}$ and longitude = $88^{\circ} 20^{\prime} \mathrm{E}$ ) and heavy vehicular load (HeVL) area at Budge Budge Trunk Road near Dakghar (latitude $=22^{\circ} 30^{\prime} \mathrm{N}$ and longitude $=88^{\circ} 15^{\prime} \mathrm{E}$ ). These three sampling stations were selected on the basis of moderate, high and heavy traffic density and continuous vehicular movement as per visualization. The control area was considered as time dependent vehicular movement due to less traffic density. The satellite image of the study area is shown in Fig. 1.

The plant species were selected viz. Ficus bengalensis, Ficus religiosa, Alstonia scholaris and Neolamarckia cadamba growing near roadside of above mentioned areas because these species are more common among other species. The affected leaf morphology by vehicular pollution was determined by the study of area of leaves and visible injuries on leaves randomly selected 5 trees of individual species of above mentioned areas. 


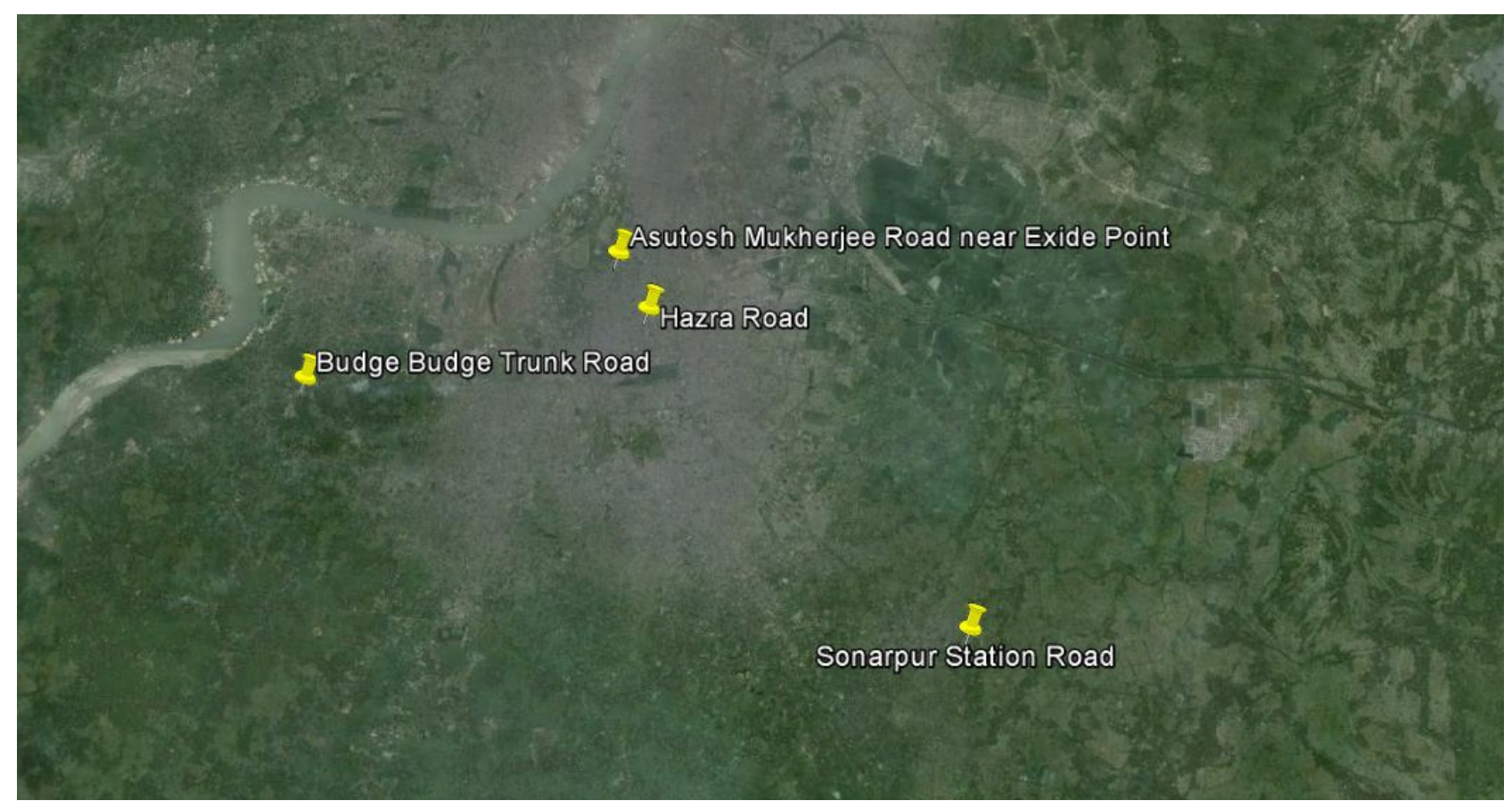

Fig. 1. Satellite image of sampling points within study area.

\section{1. Area of Leaves}

The 10 leaves were collected randomly from the selected plant species of above mentioned area. The area of leaves especially L/B (Length / Breadth) ratio of leaf (in $\mathrm{cm}$ ), was measured manually.

\section{2. Visible Injuries of Leaves}

The 50 leaves were collected randomly from the selected plant species of above mentioned area. Individual leaf was cleaned properly in running water and soaked with blotting paper. The visible injuries (in \%) of leaves viz. necrosis, cholorosis, pigmentation and burning were visualized and recorded.

\section{3. Statistical analysis}

All the mean values of data were analyzed to determine statistically significant differences between experimental and control groups by using Student's t-test at 0.05 level (Armitage and Berry, 1994).

\section{RESULTS}

The present results clearly indicate that vehicular air pollution brought significant changes in foliar morphology of four plant species viz. Ficus bengalensis, Ficus religiosa, Alstonia scholaris and Neolamarckia cadamba especially on L/B ratio (Table 1) and visible injuries in leaves (Table 2a and b; Fig. 2 and 3). The visible injuries such as pigmentation, chlorosis, necrosis and burning of leaves of four selected species is documented in Fig. 4, 5, 6 and 7. 
Table 1. Length and breadth $(\mathrm{cm})$ and L/B ratio $(\mathrm{cm})$ of leaf of selected plant species of vehicular air pollution exposed area compared to control area $(* \mathrm{P}<0.001, * * \mathrm{P}<0.01, * * * \mathrm{P}<0.05)$.

\begin{tabular}{|c|c|c|c|c|}
\hline$\omega$ & 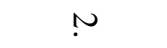 & $\vdash$ & \multicolumn{2}{|c|}{ S1. No. } \\
\hline $\begin{array}{c}\text { L/B Ratio of } \\
\text { Leaf }\end{array}$ & $\begin{array}{l}\text { Breadth of } \\
\text { Leaf }\end{array}$ & Length of Leaf & \multicolumn{2}{|c|}{ Parameters } \\
\hline $\begin{array}{l}1.70 \\
\pm 0.4\end{array}$ & $\begin{array}{l}5.41 \\
\pm 1.4\end{array}$ & $\begin{array}{l}9.40 \\
\pm 0.3\end{array}$ & 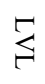 & \multirow{4}{*}{ 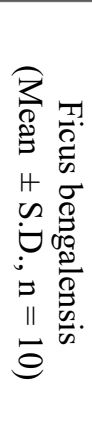 } \\
\hline $\begin{array}{c}1.74 \\
\pm 0.05\end{array}$ & $\begin{array}{c}5.5 \\
\pm 1.7\end{array}$ & $\begin{array}{l}9.44 \\
\pm 2.2\end{array}$ & 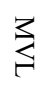 & \\
\hline $\begin{array}{l}1.56 \\
\pm 0.1\end{array}$ & $\begin{array}{c}10.6^{*} \\
\pm 2.5\end{array}$ & $\begin{array}{c}16.6^{*} \\
\pm 2.0\end{array}$ & $\lesssim$ & \\
\hline $\begin{array}{l}2.72 * \\
\pm 0.07\end{array}$ & $\begin{array}{c}7.46^{* *} \\
\pm 1.5\end{array}$ & $\begin{array}{c}19.64 * \\
\pm 3.6\end{array}$ & $\stackrel{\stackrel{T}{Q}}{2}$ & \\
\hline $\begin{array}{c}1.57 \\
\pm 0.06\end{array}$ & $\begin{array}{c}10.65 \\
\pm 0.2\end{array}$ & $\begin{array}{c}16.68 \\
\pm 0.3\end{array}$ & 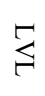 & \multirow{4}{*}{ 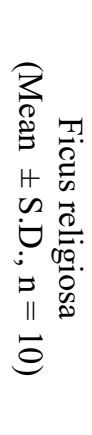 } \\
\hline $\begin{array}{l}1.96 \\
\pm 0.1\end{array}$ & $\begin{array}{l}7.49 * \\
\pm 1.8\end{array}$ & $\begin{array}{l}14.51 \\
\pm 4.2\end{array}$ & 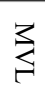 & \\
\hline $\begin{array}{l}1.73 * \\
\pm 0.1\end{array}$ & $\begin{array}{c}8.54 * * \\
\pm 2.6\end{array}$ & $\begin{array}{c}14.65 \\
\pm 3.9\end{array}$ & $\stackrel{1}{2}$ & \\
\hline $\begin{array}{l}1.63 * * \\
\pm 0.01\end{array}$ & $\begin{array}{l}9.25^{*} \\
\pm 0.07\end{array}$ & $\begin{array}{c}15.08 \\
\pm 3.4\end{array}$ &  & \\
\hline $\begin{array}{l}4.16 \\
\pm 3.4\end{array}$ & $\begin{array}{l}4.45 \\
\pm 0.2\end{array}$ & $\begin{array}{c}18.37 \\
\pm 0.5\end{array}$ & 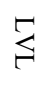 & \multirow{4}{*}{ 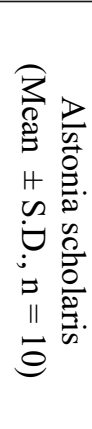 } \\
\hline $\begin{array}{c}1.75 * * * \\
\pm 0.3\end{array}$ & $\begin{array}{c}5.50 * \\
\pm 1.1\end{array}$ & $\begin{array}{c}9.44^{*} \\
\pm 2.2\end{array}$ & 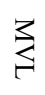 & \\
\hline $\begin{array}{c}3.09 \\
\pm 0.03\end{array}$ & $\begin{array}{c}4.70 * * \\
\pm 0.2\end{array}$ & $\begin{array}{c}14.8 * * \\
\pm 3.2\end{array}$ & 这 & \\
\hline $\begin{array}{l}3.64 \\
\pm 0.6\end{array}$ & $\begin{array}{c}3.35^{*} \\
\pm 0.5\end{array}$ & $\begin{array}{c}11.84 * \\
\pm 3.8\end{array}$ & $\stackrel{\sqrt[T]{2}}{2}$ & \\
\hline $\begin{array}{c}2.0 \\
\pm 0.09\end{array}$ & $\begin{array}{l}9.45 \\
\pm 0.8\end{array}$ & $\begin{array}{c}18.95 \\
\pm 2.5\end{array}$ & 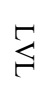 & \multirow{4}{*}{ 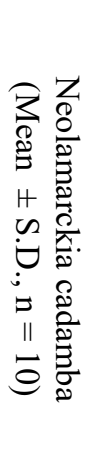 } \\
\hline $\begin{array}{c}1.97 \\
\pm 0.02\end{array}$ & $\begin{array}{c}12.98^{*} \\
\pm 2.5\end{array}$ & $\begin{array}{c}25.32 * * \\
\pm 4.7\end{array}$ & 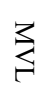 & \\
\hline $\begin{array}{l}1.77 * \\
\pm 0.1\end{array}$ & $\begin{array}{l}8.49 \\
\pm 3.0\end{array}$ & $\begin{array}{c}14.97 * * \\
\pm 3.7\end{array}$ & 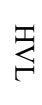 & \\
\hline $\begin{array}{l}2.43 * \\
\pm 0.06\end{array}$ & $\begin{array}{c}10.32 * * \\
\pm 0.1\end{array}$ & $\begin{array}{c}25.10^{*} \\
\pm 0.3\end{array}$ & $\stackrel{\frac{\pi}{2}}{2}$ & \\
\hline
\end{tabular}


Table 2a. Leaf visible injuries two plant species Ficus bengalensis and Ficus religiosa of vehicular air pollution exposed area compared to control area.

\begin{tabular}{|c|c|c|c|c|c|c|c|c|c|c|c|c|c|c|c|c|c|c|c|c|c|c|c|c|c|c|c|c|c|c|c|c|}
\hline \multirow{4}{*}{$\begin{array}{l}\frac{\tilde{d}}{\tilde{d}} \\
\frac{\vec{d}}{\tilde{\theta}} \\
\dot{\dot{z}}\end{array}$} & \multicolumn{32}{|c|}{ Visible injuries } \\
\hline & \multicolumn{16}{|c|}{ Ficus bengalensis (in \%) } & \multicolumn{16}{|c|}{ Ficus religiosa (in \%) } \\
\hline & \multicolumn{4}{|c|}{ LVL } & \multicolumn{4}{|c|}{ MVL } & \multicolumn{4}{|c|}{ HVL } & \multicolumn{4}{|c|}{$\mathrm{HeVL}$} & \multicolumn{4}{|c|}{ LVL } & \multicolumn{4}{|c|}{ MVL } & \multicolumn{4}{|c|}{ HVL } & \multicolumn{4}{|c|}{$\mathrm{HeVL}$} \\
\hline & $\begin{array}{c}\mathbf{P} \\
55\end{array}$ & $\begin{array}{c}\mathrm{C} \\
\mathbf{3 3} \\
\end{array}$ & $\begin{array}{c} \\
18 \\
\end{array}$ & $\begin{array}{c}B \\
16 \\
\end{array}$ & $\begin{array}{c}\mathbf{P} \\
\mathbf{6 2} \\
\end{array}$ & $\begin{array}{c}\mathbf{C} \\
\mathbf{6 6} \\
\end{array}$ & \begin{tabular}{|c|}
$\mathbf{N}$ \\
22 \\
\end{tabular} & $\begin{array}{c}\text { B } \\
\mathbf{2 0} \\
\end{array}$ & 78 & 36 & \begin{tabular}{|c|}
$\mathbf{N}$ \\
$\mathbf{9 8}$ \\
\end{tabular} & \begin{tabular}{c|} 
B \\
92
\end{tabular} & \begin{tabular}{c|}
$\mathbf{P}$ \\
$\mathbf{8 0}$
\end{tabular} & $\begin{array}{c}\mathbf{C} \\
58 \\
\end{array}$ & $\begin{array}{l}\mathbf{N} \\
86 \\
\end{array}$ & 74 & 46 & $\begin{array}{c}C \\
18 \\
\end{array}$ & $\begin{array}{c} \\
\mathbf{1 4} \\
\end{array}$ & \begin{tabular}{|c|c|} 
26 \\
26 \\
\end{tabular} & $\begin{array}{c}P \\
40 \\
\end{array}$ & $\begin{array}{c}C \\
60 \\
\end{array}$ & $\begin{array}{l}\mathbf{N} \\
\mathbf{1 8}\end{array}$ & $\begin{array}{c}\text { B } \\
34 \\
\end{array}$ & $\begin{array}{c}P \\
64 \\
\end{array}$ & $\begin{array}{c}C \\
86\end{array}$ & $\begin{array}{c} \\
22 \\
\end{array}$ & \begin{tabular}{|c|} 
B \\
$\mathbf{2 2}$ \\
\end{tabular} & \begin{tabular}{|c|}
$\mathbf{P}$ \\
$\mathbf{9 6}$ \\
\end{tabular} & $\begin{array}{c}C \\
21 \\
\end{array}$ & \begin{tabular}{|c|}
$\mathbf{N}$ \\
$\mathbf{9 8}$ \\
\end{tabular} & $\begin{array}{c}\text { B } \\
94 \\
\end{array}$ \\
\hline 1 & - & + & - & + & + & + & + & + & + & + & + & + & + & - & + & - & + & - & + & - & + & + & - & + & + & + & + & + & + & + & + & + \\
\hline 2 & + & + & - & - & + & + & + & - & + & + & + & + & + & - & + & - & + & - & + & + & + & + & - & + & + & + & - & - & + & + & + & + \\
\hline 3 & - & + & - & - & + & + & + & + & + & + & + & - & + & - & + & + & + & - & - & + & + & + & - & + & + & + & - & - & + & + & + & + \\
\hline 4 & + & + & - & - & + & - & + & - & + & + & + & + & + & - & + & + & + & - & - & + & + & + & - & + & + & + & - & - & + & + & + & - \\
\hline 5 & - & - & - & - & + & - & + & - & + & + & - & + & + & - & + & + & + & - & + & + & + & + & - & + & + & + & - & - & - & + & + & + \\
\hline 6 & - & - & - & - & + & - & + & - & + & + & + & + & + & + & + & - & + & - & - & - & - & + & - & + & + & + & + & + & + & - & + & + \\
\hline 7 & - & - & - & - & + & - & + & - & + & + & + & + & - & + & + & + & + & - & - & + & - & + & - & + & + & + & + & + & + & - & + & + \\
\hline 8 & - & - & - & - & + & + & - & - & + & + & + & + & + & + & + & + & + & - & - & - & - & + & - & - & + & + & + & + & + & - & + & - \\
\hline 9 & - & - & - & - & + & - & - & - & - & + & + & + & + & + & + & + & + & - & - & - & - & + & + & - & + & + & - & - & + & + & + & + \\
\hline 10 & - & - & - & - & + & - & - & - & + & + & + & + & + & + & + & + & + & + & - & + & + & + & + & - & + & + & - & - & + & + & + & + \\
\hline 11 & - & - & - & - & + & - & - & - & - & + & + & + & + & + & + & + & + & - & - & - & - & + & - & + & + & + & - & - & + & + & + & + \\
\hline 12 & - & - & - & - & - & + & - & - & - & + & + & + & + & + & + & - & + & + & - & + & - & + & - & - & + & + & - & - & - & + & + & + \\
\hline 13 & + & - & - & - & + & + & - & + & - & + & + & + & + & + & + & + & + & - & - & + & - & + & - & - & - & + & - & - & + & - & + & + \\
\hline 14 & + & - & - & - & + & + & - & + & - & + & + & + & + & + & + & + & + & - & - & - & - & + & - & - & - & + & + & + & + & + & + & + \\
\hline 15 & + & + & - & - & + & + & - & - & - & + & + & + & + & - & + & + & + & - & - & + & - & + & - & - & - & + & - & - & + & + & + & + \\
\hline 16 & - & + & - & - & + & + & - & - & - & - & + & + & - & - & + & + & + & - & - & - & - & + & - & - & - & + & - & - & + & + & + & + \\
\hline 17 & - & + & - & - & + & + & - & - & - & - & + & + & + & - & + & + & + & - & - & - & - & + & - & - & + & + & - & - & + & + & + & + \\
\hline 18 & - & + & - & - & - & + & - & - & - & - & + & + & + & - & + & + & + & - & - & - & - & + & - & - & + & + & - & - & + & + & + & + \\
\hline 19 & - & - & - & - & - & + & - & + & - & - & + & - & + & - & $t$ & + & + & - & - & - & - & + & - & - & + & + & - & - & + & + & + & + \\
\hline 20 & - & + & - & - & - & - & + & _ & + & + & + & + & - & - & + & + & + & - & + & - & - & + & - & + & + & + & - & - & + & - & - & + \\
\hline 21 & - & - & - & - & + & - & + & - & + & + & + & + & + & + & + & + & + & - & - & - & + & + & - & + & - & + & - & - & + & - & + & + \\
\hline 22 & - & + & - & - & + & - & - & - & + & + & + & + & + & + & + & + & + & + & - & - & + & + & - & + & - & + & - & - & + & - & + & + \\
\hline 23 & - & + & + & - & + & + & - & - & + & + & + & + & - & + & + & + & + & + & - & - & + & + & - & + & - & + & - & - & + & - & + & + \\
\hline 24 & - & + & - & - & + & + & - & - & + & + & + & + & + & + & + & + & - & + & - & - & + & & - & - & - & + & - & - & + & - & + & + \\
\hline 25 & + & - & + & - & + & + & - & - & + & + & + & + & + & - & + & + & - & + & - & - & + & - & - & - & - & + & - & - & + & + & + & + \\
\hline 26 & + & - & - & - & + & + & - & + & + & + & + & + & + & + & + & + & - & - & - & - & + & - & - & - & - & + & - & - & + & - & + & + \\
\hline 27 & - & - & + & - & + & + & - & + & + & - & + & - & + & + & + & + & - & - & + & - & + & - & - & - & - & + & - & - & + & - & + & + \\
\hline 28 & - & - & - & - & + & + & - & + & + & - & + & + & + & + & + & + & - & - & - & - & + & - & - & - & - & + & - & - & + & - & + & + \\
\hline 29 & - & - & - & - & + & + & - & + & + & - & + & + & + & + & + & + & - & - & - & - & + & - & + & - & + & + & - & - & + & - & + & + \\
\hline 30 & - & - & - & - & + & + & - & + & + & + & + & + & - & - & - & - & - & - & - & + & + & - & + & - & + & + & - & - & + & - & + & + \\
\hline 31 & - & + & - & - & + & + & - & - & + & + & + & + & - & - & - & - & - & - & - & + & + & - & + & - & + & + & - & - & + & - & + & + \\
\hline 32 & - & + & - & - & + & + & - & - & + & - & + & + & + & - & - & - & - & - & - & + & + & - & + & - & + & + & - & - & + & - & + & + \\
\hline 33 & - & + & - & - & - & + & - & - & + & - & + & + & + & - & - & - & - & + & - & - & + & + & + & - & + & + & - & \begin{tabular}{|l|}
- \\
\end{tabular} & + & - & + & + \\
\hline 34 & + & + & - & - & - & + & - & - & + & + & + & + & + & - & + & + & - & - & - & - & - & + & + & - & + & + & - & - & - & - & + & + \\
\hline 35 & + & + & - & - & - & + & - & - & + & - & + & + & + & + & + & + & - & - & - & - & - & + & + & - & - & + & - & - & + & - & + & + \\
\hline 36 & + & - & - & - & - & + & - & - & + & + & + & + & + & + & + & + & - & - & - & - & - & + & - & + & + & + & - & - & + & + & + & + \\
\hline
\end{tabular}




\begin{tabular}{|l|c|c|c|c|c|c|c|c|c|c|c|c|c|c|c|c|c|c|c|c|c|c|c|c|c|c|c|c|c|c|c|c|}
\hline $\mathbf{3 7}$ & + & - & - & - & - & + & - & - & + & + & + & + & - & + & - & - & - & - & - & - & - & + & - & - & - & + & - & - & + & - & + & + \\
\hline $\mathbf{3 8}$ & + & - & - & - & - & + & - & - & + & - & + & + & - & + & - & + & - & - & - & - & - & - & - & - & + & - & - & - & + & - & + & + \\
\hline $\mathbf{3 9}$ & + & - & - & - & - & + & - & - & + & - & + & + & + & + & - & + & - & + & - & - & - & - & - & - & + & - & - & - & + & - & + & + \\
\hline $\mathbf{4 0}$ & + & - & - & - & - & + & - & - & + & - & + & - & + & + & + & + & - & + & + & - & - & - & - & - & + & - & - & - & + & - & - & + \\
\hline $\mathbf{4 1}$ & + & - & - & - & - & + & - & - & + & - & + & + & + & + & + & + & - & - & - & + & - & - & - & - & + & + & - & - & + & - & + & + \\
\hline $\mathbf{4 2}$ & - & - & - & - & - & - & - & - & + & - & + & + & + & + & + & + & - & - & - & - & - & + & - & - & + & + & - & - & + & - & + & - \\
\hline $\mathbf{4 3}$ & - & - & - & - & + & - & - & - & + & - & + & + & + & - & + & - & - & - & - & - & - & + & - & - & + & + & - & - & + & - & + & + \\
\hline $\mathbf{4 4}$ & - & - & - & + & + & - & - & - & + & - & + & + & + & + & + & - & - & - & - & - & - & - & - & + & + & + & - & - & + & - & + & + \\
\hline $\mathbf{4 5}$ & - & - & - & + & + & + & - & - & + & - & + & + & + & - & + & - & - & - & - & - & - & - & - & - & + & + & + & + & + & - & + & + \\
\hline $\mathbf{4 6}$ & - & - & - & + & - & - & + & - & + & - & + & + & + & + & + & - & - & - & - & - & + & - & - & - & + & - & + & + & + & - & + & + \\
\hline $\mathbf{4 7}$ & + & - & + & + & - & - & + & - & + & - & + & + & - & + & + & + & - & - & - & - & - & - & - & - & - & - & + & + & + & - & + & + \\
\hline $\mathbf{4 8}$ & + & - & - & + & - & + & + & - & + & - & + & + & + & + & + & + & - & - & + & - & - & - & - & + & - & - & + & + & + & -- & + & + \\
\hline $\mathbf{4 9}$ & + & - & - & + & - & - & + & - & + & - & + & + & + & - & + & + & - & - & - & - & - & - & - & + & - & + & + & + & + & - & + & + \\
\hline $\mathbf{5 0}$ & + & - & + & + & - & - & - & - & + & - & + & + & + & - & + & + & - & - & - & - & - & - & - & + & - & + & + & + & + & - & + & + \\
\hline
\end{tabular}

$\mathrm{P}=$ Pigmentation-; $\mathrm{C}=$ Chlorosis $; \mathrm{N}=$ Necrosis; $\mathrm{B}=$ Burning; $(+)=$ Injuries Present $(-)=$ Injuries Absent

Table 2b. Leaf visible injuries two plant species Alstonia scholaris and Neolamarckia cadamba of vehicular air pollution exposed area compared to control area.

\begin{tabular}{|c|c|c|c|c|c|c|c|c|c|c|c|c|c|c|c|c|c|c|c|c|c|c|c|c|c|c|c|c|c|c|c|c|c|}
\hline \multirow{4}{*}{ 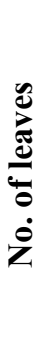 } & \multicolumn{33}{|c|}{ Visible injuries } \\
\hline & \multicolumn{16}{|c|}{ Alstonia scholaris (in \%) } & \multicolumn{17}{|c|}{ Neolamarckia cadamba (in \%) } \\
\hline & \multicolumn{4}{|c|}{ LVL } & \multicolumn{4}{|c|}{ MVL } & \multicolumn{4}{|c|}{ HVL } & \multicolumn{4}{|c|}{$\mathrm{HeVL}$} & \multicolumn{5}{|c|}{ LVL } & \multicolumn{4}{|c|}{ MVL } & \multicolumn{4}{|c|}{ HVL } & \multicolumn{4}{|c|}{$\mathrm{HeVL}$} \\
\hline & $\begin{array}{c}\mathrm{P} \\
56\end{array}$ & $\begin{array}{c}\mathrm{C} \\
06\end{array}$ & $\begin{array}{c}\mathrm{N} \\
01\end{array}$ & $\begin{array}{c}\text { B } \\
08\end{array}$ & $\begin{array}{c}\mathrm{P} \\
72\end{array}$ & $\begin{array}{c}\mathrm{C} \\
14\end{array}$ & $\begin{array}{l}\mathrm{N} \\
15\end{array}$ & $\begin{array}{c}\text { B } \\
36\end{array}$ & $\begin{array}{c}P \\
62\end{array}$ & $\begin{array}{c}C \\
40\end{array}$ & $\begin{array}{c}\mathrm{N} \\
80\end{array}$ & $\begin{array}{l}\mathrm{B} \\
50\end{array}$ & $\begin{array}{c}\mathrm{P} \\
84\end{array}$ & $\begin{array}{c}\text { C } \\
30\end{array}$ & $\begin{array}{l}\mathrm{N} \\
54\end{array}$ & $\begin{array}{c}\text { B } \\
42\end{array}$ & $\begin{array}{c}P \\
56\end{array}$ & $\begin{array}{c}\mathrm{C} \\
04\end{array}$ & $\begin{array}{l}1 \\
0\end{array}$ & & $\begin{array}{c}B \\
0\end{array}$ & $\begin{array}{l}\mathrm{P} \\
74\end{array}$ & $\begin{array}{c}\mathrm{C} \\
38\end{array}$ & $\begin{array}{c}\mathrm{N} \\
32\end{array}$ & $\begin{array}{c}\mathrm{B} \\
30\end{array}$ & $\begin{array}{c}\mathrm{P} \\
62\end{array}$ & $\begin{array}{c}\mathrm{C} \\
36\end{array}$ & $\begin{array}{c}\mathrm{N} \\
24\end{array}$ & $\begin{array}{c}\mathrm{B} \\
22\end{array}$ & $\begin{array}{c}\mathrm{P} \\
90\end{array}$ & $\begin{array}{c}C \\
76\end{array}$ & $\begin{array}{c}\mathrm{N} \\
40\end{array}$ & $\begin{array}{c}\text { B } \\
78\end{array}$ \\
\hline 1 & - & + & - & - & + & - & - & - & . & + & + & - & + & - & - & - & - & - & . & & - & + & + & - & + & + & + & + & - & + & + & + & + \\
\hline 2 & - & + & - & - & - & - & + & - & + & + & + & + & + & - & - & - & - & - & . & & - & + & + & - & + & + & + & - & + & + & + & + & + \\
\hline 3 & - & - & - & - & - & - & - & - & + & + & + & + & + & + & + & - & - & - & . & & - & + & + & - & + & + & + & - & + & + & + & + & + \\
\hline 4 & + & - & - & + & - & - & - & - & + & + & + & + & + & - & + & - & + & - & . & & - & + & + & - & + & + & - & - & + & + & + & + & + \\
\hline 5 & + & - & + & - & - & - & - & - & - & + & + & - & + & + & + & - & - & - & . & & - & + & + & - & + & + & - & - & - & + & + & + & - \\
\hline 6 & - & - & - & - & - & - & - & - & - & + & + & + & + & - & - & + & - & - & . & & - & + & + & - & + & + & - & - & - & + & + & + & - \\
\hline 7 & - & - & - & - & - & - & + & + & - & + & - & + & - & + & - & + & + &  & . & & - & + & - & - & + & + & - & - & - & + & + & - & - \\
\hline 8 & - & - & - & + & - & - & - & + & - & + & + & - & + & + & - & - & + & - & . & & - & + & - & - & + & + & + & - & - & + & + & - & - \\
\hline 9 & + & - & - & - & - & - & + & + & - & - & + & - & + & + & - & - & + & - & . & & - & + & - & - & + & + & + & + & + & + & + & + & + \\
\hline 10 & - & - & - & - & - & - & + & - & - & - & + & - & + & + & - & - & & - & . & & - & + & - & - & + & + & - & + & + & + & - & + & - \\
\hline 11 & - & - & - & - & - & - & + & - & + & - & - & + & + & - & + & - & + & - & . & & - & + & - & - & + & + & - & - & - & + & - & + & - \\
\hline 12 & - & - & - & - & - & - & - & - & + & + & - & + & + & - & + & + & + & - & . & & - & + & - & - & + & + & - & + & + & + & - & + & - \\
\hline 13 & - & - & - & - & - & - & + & - & + & + & - & + & + & - & + & + & + & - & . & & - & $=$ & - & - & - & + & + & + & - & + & + & + & - \\
\hline 14 & - & - & - & - & - & - & + & - & + & + & - & + & + & - & + & + & . & 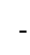 & . & & - & + & + & - & - & + & - & - & - & + & + & + & - \\
\hline 15 & - & - & - & - & - & - & - & - & + & + & - & + & + & - & + & + & + & - & & & - & + & + & - & - & + & + & - & - & + & + & + & - \\
\hline
\end{tabular}




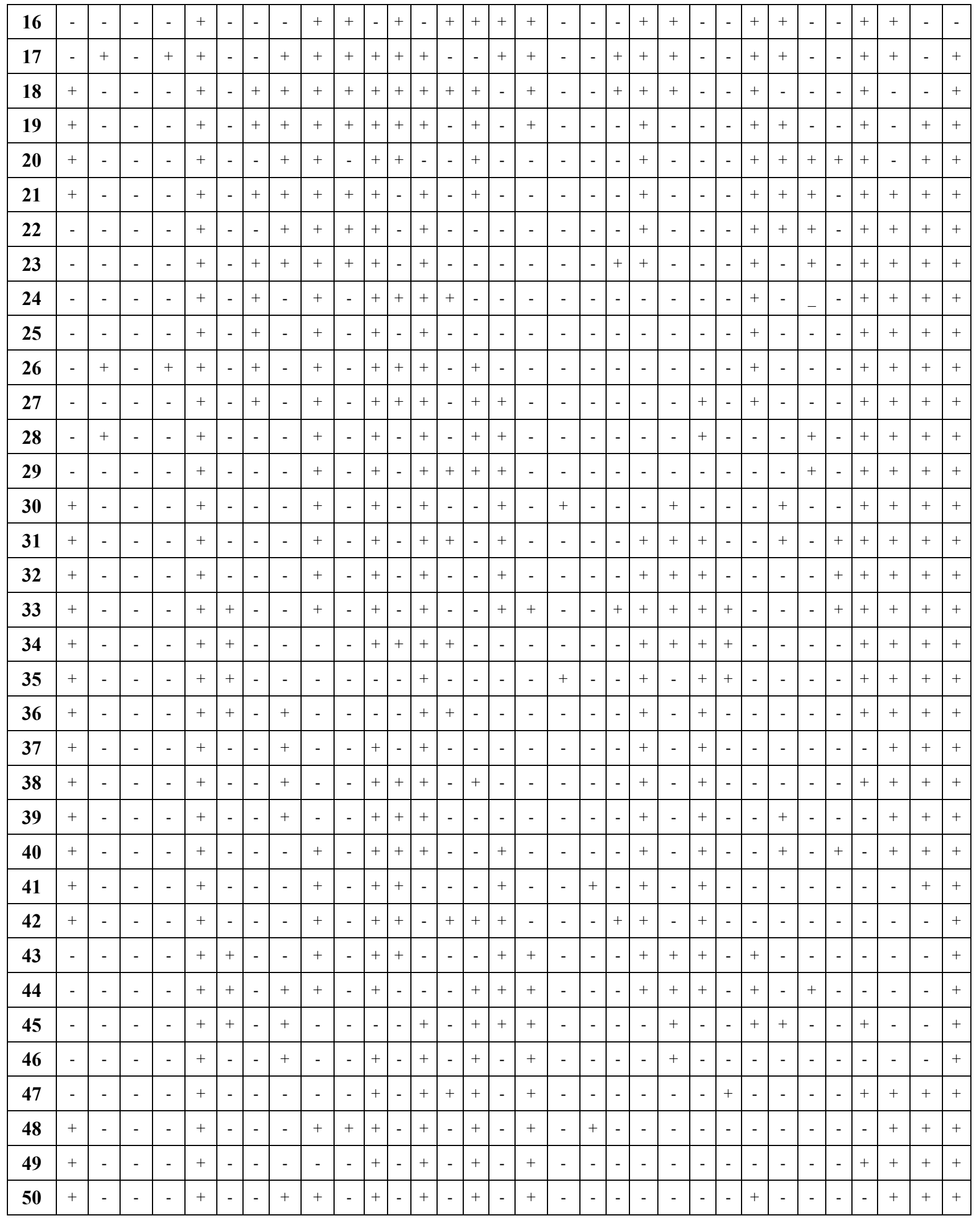

$\mathrm{P}=$ Pigmentation-; $\mathrm{C}=$ Chlorosis; $\mathrm{N}=$ Necrosis; $\mathrm{B}=$ Burning; $(+)=$ Injuries Present; $(-)=$ Injuries Absent 


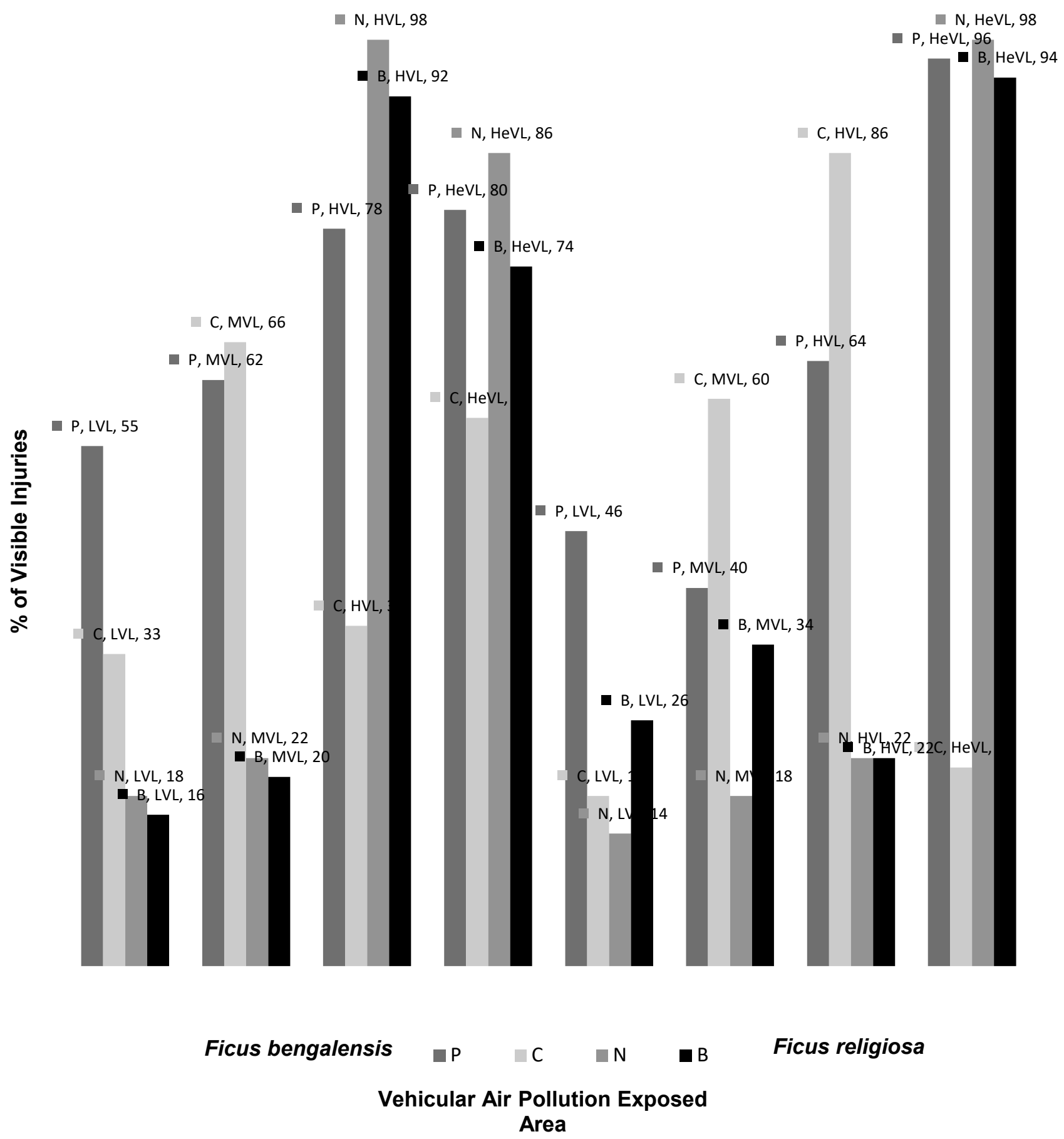

Fig. 2. Bar diagram showing \% visible injuries in leaf of Ficus bengalensis and Ficus religiosa. 




Fig. 3. Bar diagram showing \% visible injuries in leaf of Alstonia scholaris and Neolamarckia cadamba. 


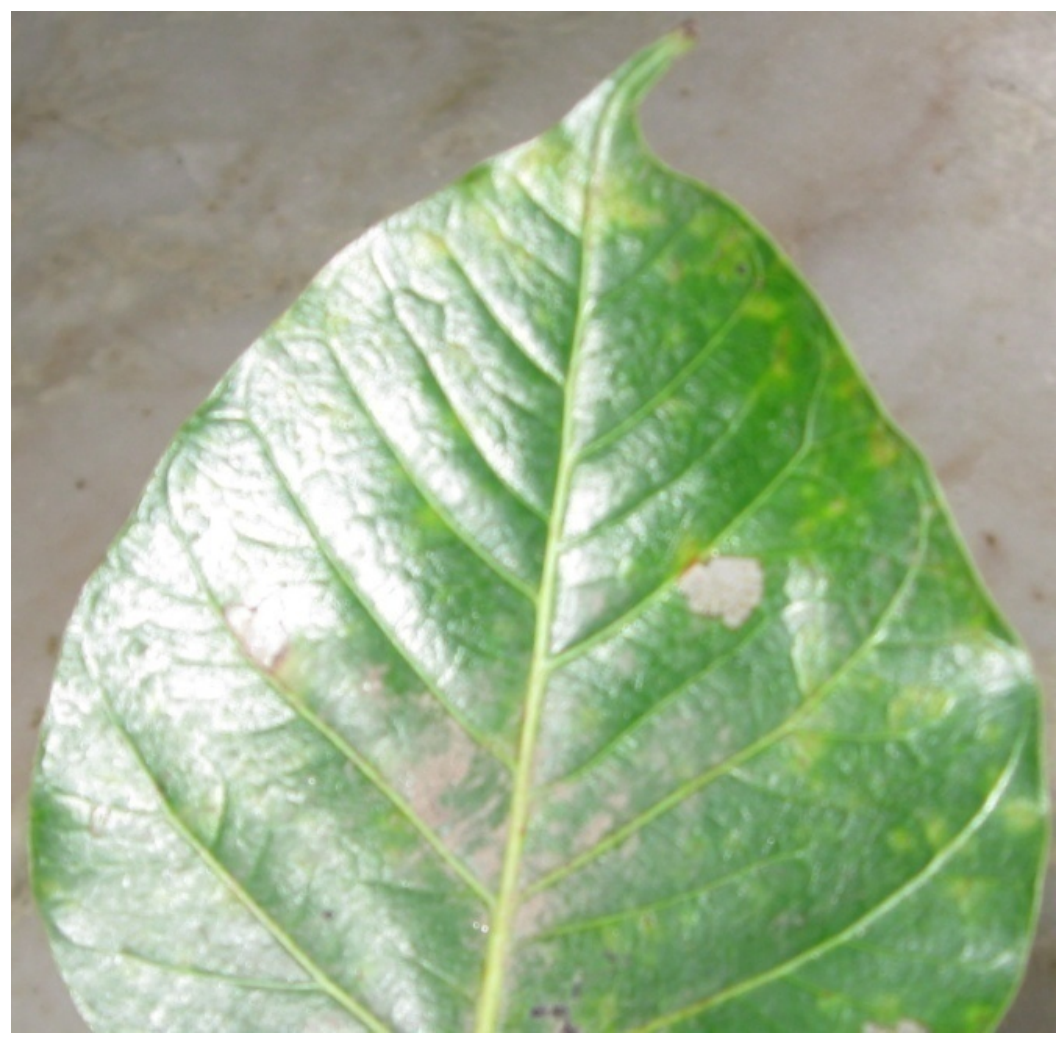

Fig. 4. Pigmentation and necrosis in leaf of Ficus religiosa.

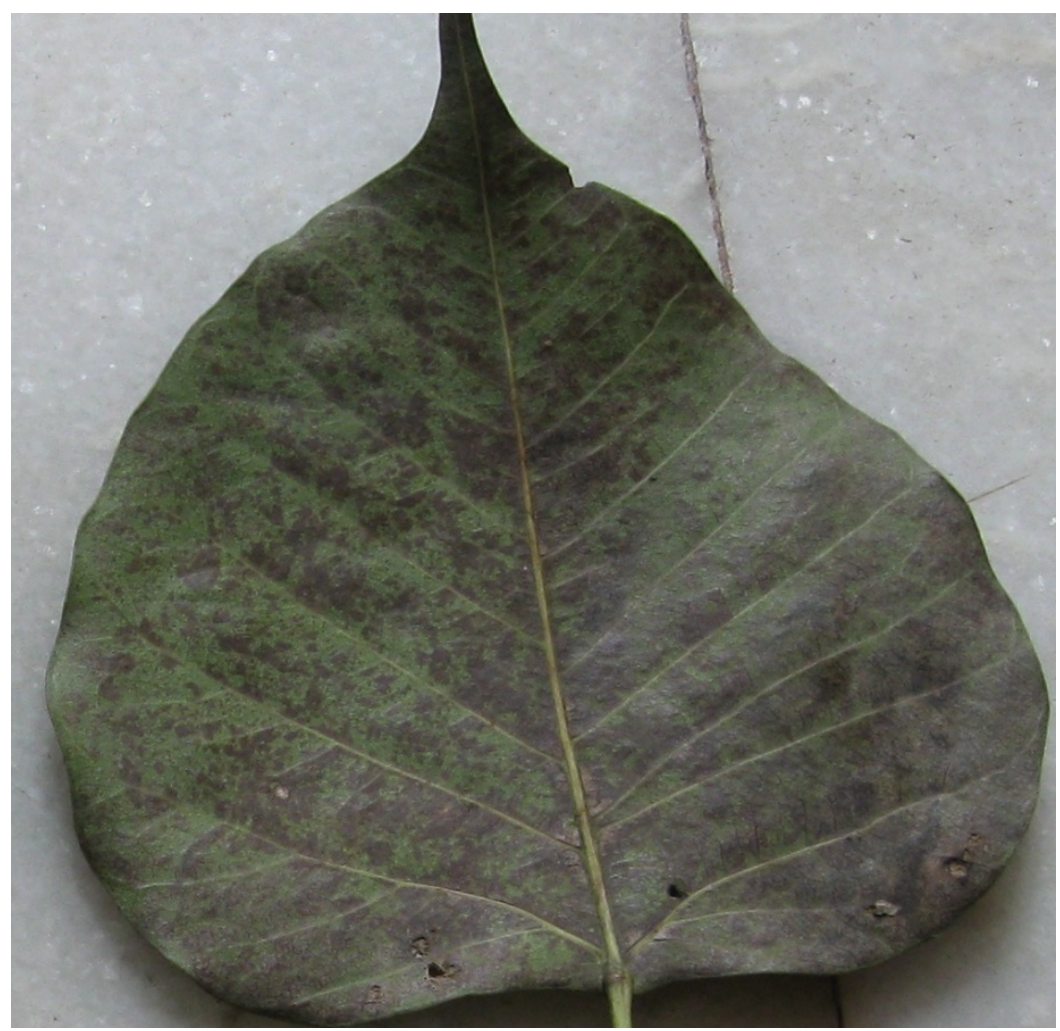

Fig. 5. Necrosis in leaf of Ficus religiosa. 


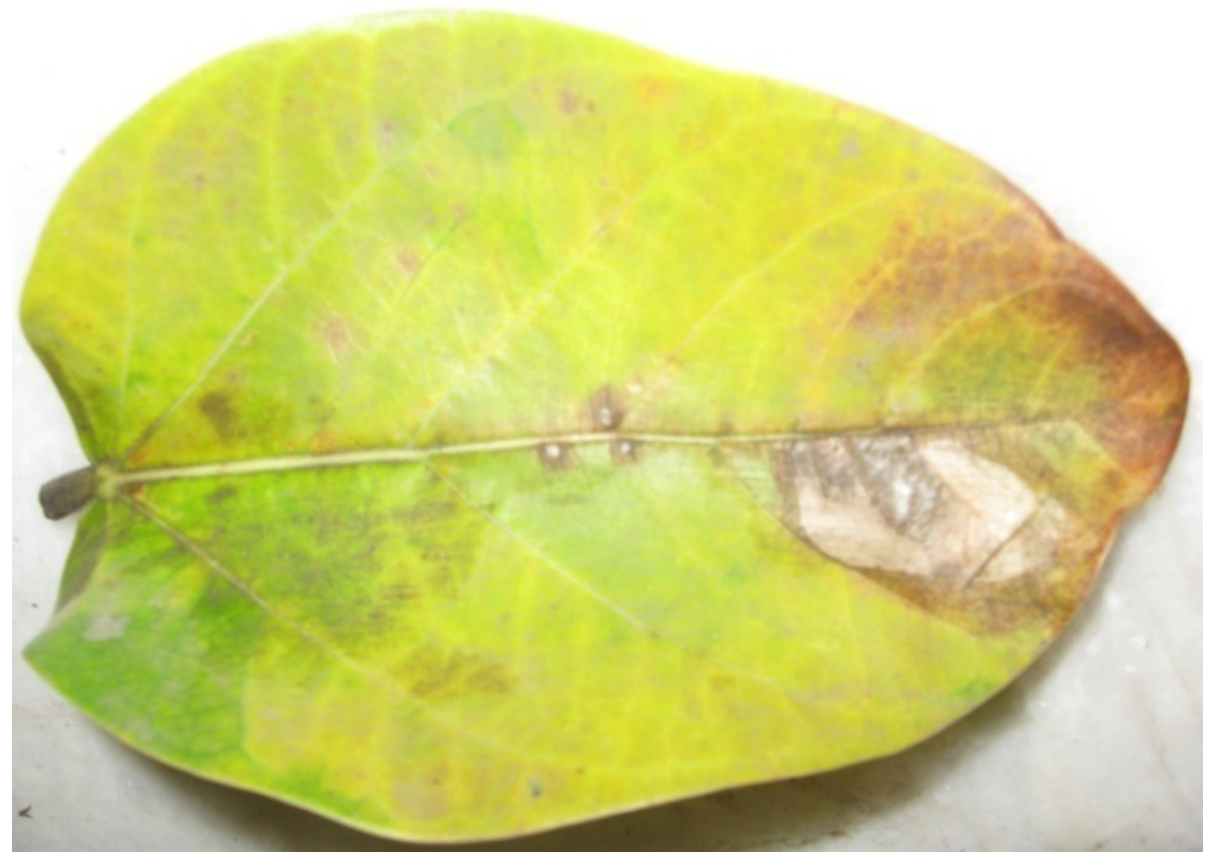

Fig. 6. Cholorosis and necrosis in leaf of Ficus bengalensis.

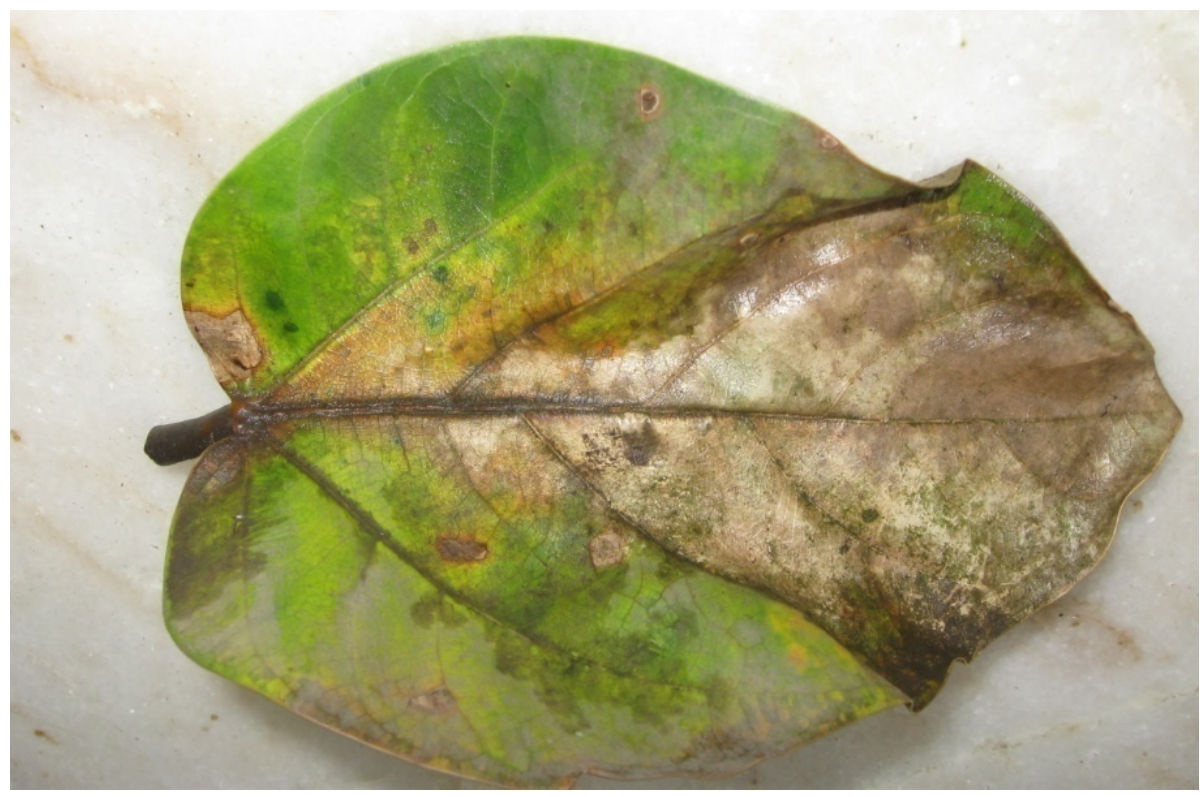

Fig. 7. Pigmentation, burning and necrosis in leaf of Ficus bengalensis.

In all experimental sites such as MVL, HVL and HeVL, the extra growth and reduction pattern significantly $(\mathrm{P}<0.001,0.01$ or 0.05$)$ observed when compared to control site (LVL) for $\mathrm{L}, \mathrm{B}$ and $\mathrm{L} / \mathrm{B}$ ratio. The visible injuries of leaves were also observed in increasing trends.

There was an increasing and decreasing tends in all four plant species at all three vehicular emission exposed sites (MVL, HVL and HeVL) compared to control site (LVL) is shown in Table 1. The L/B ratio of leaf was observed decreasing trend at a significance level of $\mathrm{P}<0.05$ in Alstonia scholaris at MVL area and $\mathrm{P}<0.001$ in Neolamarckia cadamba at 
HVL area but somehow statistically significance level $(\mathrm{P}<0.001)$ of increasing trend for HeVL while it was observed an increasing trends both in Ficus bengalensis and Ficus religiosa but the statistically significant data $(\mathrm{P}<0.001)$ were only shown in Ficus bengalensis at HeVL and for Ficus religiosa at HVL $(\mathrm{P}<0.001)$ and at HeVL $(\mathrm{P}<0.01)$ when compared to control area at LVL.

Although the breadth of leaves found a statistically significant reduction at MVL and HeVL $(\mathrm{P}<0.001)$ and also at HVL $(\mathrm{P}<0.01)$ in Ficus religiosa but in Ficus bengalensis, Alstonia scholaris and Neolamarckia cadamba showed an extra growth at HVL, MVL and HeVL site when compared with LVL site. The statistical significance data were observed at HVL $(\mathrm{P}<0.001)$ and HeVL $(\mathrm{P}<0.01)$ in Ficus bengalensis, at only MVL $(\mathrm{P}<0.05)$ in Alstonia scholaris and at MVL $(\mathrm{P}<0.001)$ and at HeVL $(\mathrm{P}<0.01)$ in Neolamarckia cadamba, while the length of the leaf in Ficus religiosa minor reduction and in Alstonia scholaris was showed significantly major reduction at MVL and HeVL $(\mathrm{P}<0.001)$ whereas at HVL $(\mathrm{P}<0.01)$ but an extra growth in Ficus bengalensis the significant data only at HVL and HeVL $(\mathrm{P}<0.001)$ and in Neolamarckia cadamba the level of significance were showed at HeVL $(\mathrm{P}<0.001)$, HVL $(\mathrm{P}<0.01)$ and MVL $(\mathrm{P}<0.05)$ area when compared to control area (LVL).

The visible injuries were observed majorly in all vehicular exposed area when compared to control area (Table 2a and b; Fig. 2 and 3). It was also observed that the injuries maximum necrosis and chlorosis followed by pigmentation and burning but injuries are species specific (Fig. 4, 5, 6 and 7). It was also noted that all above-mentioned parameters were present in same leaf and/or any one parameter was only observed. The data were scored and recorded on individual parameter basis in each selected species.

In case of the Ficus bengalensis necrosis was higher (98\%) in HVL followed by $86 \%$ in HeVL and $22 \%$ in MVL when compared with LVL (18\%) while chlorosis was higher (58 $\%$ ) in HeVL followed by $66 \%$ in MVL and $36 \%$ in HVL when compared with LVL (33\%). The pigmentation and burning were observed higher ( $80 \%$ in HeVL and $92 \%$ in HVL), moderate $(78 \%$ in HVL and $74 \%$ in HeVL) and the MVL area was shown very close differences as $62 \%$ and $20 \%$ when compared with LVL (55\% and $16 \%)$.

In case of the Ficus religiosa necrosis was higher (98\%) in HeVL followed by $22 \%$ in HVL and $18 \%$ in MVL when compared with LVL (14\%) while chlorosis was higher (86\%) in HVL followed by $60 \%$ in MVL and shown less differences of $21 \%$ in HeVL when compared with LVL (18\%). The pigmentation and burning were observed higher $(96 \%$ and $94 \%$ ) only in HeVL, moderate and less pigmentation were $64 \%$ in HVL and $40 \%$ in MVL when compared with LVL (46\%) while the moderate burning (34\%) was in MVL and low burning (22\%) in HVL area was shown low value when compared with LVL (46\%).

In case of the Alstonia scholaris necrosis was higher (80\%) in HVL followed by $54 \%$ in $\mathrm{HeVL}$ and $15 \%$ in MVL when compared with LVL $(01 \%)$ while chlorosis was higher (40 $\%$ ) in HVL followed by $30 \%$ in HeVL and shown low $21 \%$ in HeVL when compared with LVL (18\%). The pigmentation was observed higher (84\% and $72 \%$ ) in HeVL and MVL and lower value $62 \%$ in HVL was closely related when compared with LVL (56\%) while the higher value $(50 \%)$ of burning was in HVL, followed by $42 \%$ in $\mathrm{HeVL}, 36 \%$ in MVL when compared with LVL $(08 \%)$.

In case of the Neolamarckia cadamba necrosis was higher (40\%) in HeVL followed by $32 \%$ in MVL and $24 \%$ in HVL when compared with LVL (04\%) while chlorosis was higher $(76 \%)$ in HeVL followed by $38 \%$ in MVL and $36 \%$ in HVL when compared with LVL (04\%). The pigmentation was observed higher (90\% and $74 \%$ ) in HeVL and MVL and moderate value $62 \%$ in HVL was closely related when compared with LVL $(51 \%)$ 
while the higher value (78 \%) of burning was in HeVL, followed by $30 \%$ in MVL, $22 \%$ in HVL when compared with LVL (10\%).

\section{DISCUSSION AND CONCLOSIONS}

The present study of four common selected species viz. Ficus bengalensis, Ficus religiosa, Alstonia scholaris and Neolamarckia cadamba growing near roadside of MVL, HVL and HeVL areas when compared with control area (LVL) indicate that vehicular air pollution brought significant changes in few area on foliar morphology especially L, B and $\mathrm{L} / \mathrm{B}$ ratio of leaves and high percentage $(\%)$ of visible injuries on leaves.

The adverse impacts of air pollution on living biota with special reference to plant species have already been studied nationally and internationally. Major research works have been conducted on the physical and chemical analysis of air pollutant by using various instruments (CPCB, 2009; Citizen's Report, 2011). The effects on crops and vegetation especially leaves morphology, visible injuries, biochemical alterations etc have been done internationally (Bull and Mansfield, 1974; Husen et al., 1999; Naveed et al., 2010; Seyyed and Koochak 2011) but few works have been done in India (Tiwari et al., 2006; Joshi and Swami 2007; Tiwary et al., 2008; Saquib et al., 2010; Deepalakshmi, 2013) but many researchers have been documented on physico-chemical analysis of air pollutants, no one has tried to establish bioindicator study in relation to foliar morphology especially L, B and L/B ratio and visible injuries of leaves of four common trees near roadside at Kolkata, India.

As we know, urban air pollution is a matter of great concern $(\mathrm{Li}, 2003)$. Air pollution can directly affect plants via leaves or indirectly via soil acidification. When air pollutants exposed to ambient environment, most plants experienced physiological changes before exhibiting visible damage to leaves (Liu and Ding, 2008). Plants that are constantly exposed to environmental pollutants absorb, accumulate and integrate these pollutants into their systems. It reported that depending on their sensitivity level, plants show visible changes which would include alteration in the biochemical processes or accumulation of certain metabolites (Agbaire and Esiefarienrhe, 2009). Vegetation is an effective indicator of the overall impact of air pollution (Rai et al., 2009). Pollutants can cause leaf injury, stomatal damage, premature senescence, decrease photosynthetic activity, disturb membrane permeability and reduce growth and yield in sensitive plant species (Tiwari et al., 2006). This finding supports with evident for other researchers that selected common species were more tolerant and less tolerant located in maximum vehicular movement area (MVL, HVL and $\mathrm{HeVL}$ ) and showed increasing as well as decreasing morphological damages by air pollution when compared to control area (LVL).

Table 1 is shown an increasing and decreasing tends in all four plant species at all three vehicular emission exposed sites (MVL, HVL and HeVL) compared to control site (LVL). The L/B ratio of leaf was observed decreasing trend at a significance level of $\mathrm{P}<0.05$ in Alstonia scholaris at MVL area and $\mathrm{P}<0.001$ in Neolamarckia cadamba at HVL area but somehow statistically significance level $(\mathrm{P}<0.001)$ of increasing trend for HeVL while it was observed an increasing trends both in Ficus bengalensis and Ficus religiosa but the statistically significant data $(\mathrm{P}<0.001)$ were only shown in Ficus bengalensis at HeVL and for Ficus religiosa at HVL $(\mathrm{P}<0.001)$ and at HeVL $(\mathrm{P}<0.01)$ when compared to control area at LVL. Although the breadth of leaves found a statistically significant reduction at MVL and HeVL $(\mathrm{P}<0.001)$ and also at HVL $(\mathrm{P}<0.01)$ in Ficus religiosa but in Ficus bengalensis, Alstonia scholaris and Neolamarckia cadamba showed an extra growth at HVL, 
MVL and HeVL site when compared with LVL site. The statistical significance data were observed at HVL $(\mathrm{P}<0.001)$ and HeVL $(\mathrm{P}<0.01)$ in Ficus bengalensis, at only MVL $(\mathrm{P}<$ $0.05)$ in Alstonia scholaris and at MVL $(\mathrm{P}<0.001)$ and at HeVL $(\mathrm{P}<0.01)$ in Neolamarckia cadamba, while the length of the leaf in Ficus religiosa minor reduction and in Alstonia scholaris was showed significantly major reduction at MVL and HeVL $(\mathrm{P}<0.001)$ whereas at HVL $(\mathrm{P}<0.01)$ but an extra growth in Ficus bengalensis the significant data only at HVL and HeVL $(\mathrm{P}<0.001)$ and in Neolamarckia cadamba the level of significance were showed at HeVL $(\mathrm{P}<0.001)$, HVL $(\mathrm{P}<0.01)$ and MVL $(\mathrm{P}<0.05)$ area when compared to control area (LVL). It indicates species specific and automobile air pollutants effects that resemblances the previous study (Gummani et al., 1991; Kulshreshtha et al. 1994a and b; Deepalakshmi, 2013). Overall the present study supports with other research work on different plant species exposed to gaseous and particulate pollutants. Jain and Sreelatha (2006), and Tiwari et al. (2008) have also reported reduction in leaf area of C. siamea and due to dust pollution. It appears in present study that may SPM along with air pollutants like $\mathrm{O}_{3}, \mathrm{SO}_{2}$ and NOx, PAN and also have more damaging effect on leaves (Joshi and Swami 2007; Deepalakshmi, 2013), as a result there was more reduction in leaf area, which supports the present study. But still the growth of leaves is not clear. The growth may be due to the accumulation of air pollutants, generation of more waxy coatings and by rapid cell elongation process.

The effects of air pollution on plants include mottled foliage, "burning" at leaf tips or margins, twig dieback, stunted growth, premature leaf drop, delayed maturity, abortion or early drop of blossoms, and reduced yield or quality. In general, there are three types of visible injury to plants have been established, firstly collapse of leaf tissue with the development of necrotic patterns, secondly yellowing or other color changes, and thirdly alterations in growth or premature loss of foliage (Fig. 4, 5, 6 and 7). But visible injuries from air pollution can be confused with the symptoms caused by fungi, bacteria, viruses, nematodes, insects, nutritional deficiencies and toxicities, and the adverse effects of temperature, wind, and water. The present study has emphasized with previous research works on monitoring and physico-chemical analysis of air pollutants especially particulates and gaseous pollutants in and around Kolkata city (Citizen report, 2011).

The present results of visible injuries were established that the percentage (\%) of necrosis, chlorosis, pigmentation and burning in leaves of selected species have potent accumulation capacity as well as can protect air pollution easily as tolerant species of three out of four species viz. Ficus bengalensis, Alstonia scholaris and Neolamarckia cadamba while one spices namely Ficus religiosa is a less tolerant species to vehicular air pollution at all exposed area when compared to control area (Table 2a and b; Fig. 2 and 3). The air pollutants include gases (sulphur dioxide, nitrogen oxides, carbon monoxides, hydrocarbons, ozone etc.), particulate matters (smoke, dust, fumes, aerosols, etc.), radioactive materials and many others. Air pollution may or will have harmful effects on living creatures. It may interfere with biochemical and physiological processes of plants to an extent, which ultimately leads to yield losses (Middleton et al., 1956; Heck et al., 1988). Recent trends have shown decrease in $\mathrm{SO}_{2}$ emissions, but increase in $\mathrm{NO}_{2}$ emission due to more number of automobiles. In past few decades, tropospheric $\mathrm{O}_{3}$ has been identified as a most important air pollutant of rural areas. Air pollutants produce reactive oxygen species (ROS), which adversely affect biochemical processes of plants and reduce their tolerance capacity to other stresses also. It was also reviewed that present and future trends of major gaseous pollutants emissions and their impact on crop performance (Rai et al., 2011), which supports the present data on morphological anomalies but these trees are still fighting with air pollutants. 
In the present study it was concluded that the adverse effects mainly morphological damages viz. L (Length), B (Breadth) and L/B ratio was found significantly increasing and decreasing trends and visible injuries such as necrosis, chlorosis, pigmentation and burning were observed in an increasing as well as decreasing trends by automobile air pollution in four selected common roadside plant species, which may be the effects of individual and/or combination of air pollutants though there no attempt has been made on physico-chemical properties of present air pollutants. As we know from the previous research work in Indian cities, the concentrations of phytotoxic air pollutants often exceed the toxic limits (Trivedi et al., 2003; CPCB, 2009). This study is a preliminary assessment of tolerant species that already have been used in greenbelt development to protect air pollutants as well as biological monitoring to know exact load of industrial and/or automobiles air pollution but further researches are needed in relation to biochemical and genetic damage study as well as air pollution load by using instruments. It was observed that out of four selected species Ficus bengalensis, Alstonia scholaris and Neolamarckia cadamba are more tolerant species and Ficus religiosa is a less tolerant species because of these may have fighting abilities by waxy coatings, accumulation and degradation abilities to vehicular air pollution at all exposed area when compared to control area.

\section{ACKNOWLEDGEMENT}

The authors convey their gratitude to the Department of Environmental Science, University of Calcutta, for providing the necessary infrastructure for doing this study.

\section{References}

[1] Agbaire P. O., Esiefarienrhe E. J., Appl Sci Environ Manage 13(1) (2009) 11-14.

[2] Armitage P., Berry G., Statistical methods in medical research. IIIrd Edn. ISBN 0-63203695-8, Oxford, Blackwell Scientific Publication, New York, (1994) pp. 103-115 and 207-214.

[3] Bull J. N., Mansfield T. A., Nature 250 (1974) 443.

[4] Citizen's Report, Centre of Science and Environment (2011) 1-106.

[5] CPCB, Central Pollution Control Board, New Delhi., (2009). http://www.cpcb.nic.in/bulletin/del/2009html.

[6] Deepalakshmi A. P., Ramakrishnaiah H., Ramachandra,Y. L., Radhika R. N., J Environ Sci Toxicol Food Tech 3(3) (2013) 10-14.

[7] Gummani T., Guruswamy R., Saminathan, Effect J. Swamy Bot. Club 8(3 and 4) (1991) 79-85.

[8] Heck W. W., Taylor O. C., Tingey D. T. Tingey., Applied Science (1988).

[9] Husen A., Ali S. T., Mahmooduzzafar Iqbal M., Proc. Acad. Environ. Biol. 8 (1999) 61-72.

[10] Joshi P. C., Swami A., Environmentalist 27 (2007) 365-374.

[11] Kulshreshtha K., Farrookqui A. Srivastava K. Singh S. N., Ahmed K. J., Behl H. M., J. Environ. Sci: Part-A. Envion. Sci. Engg. 29 (1994a) 300-308. 
[12] Kulshreshtha K., Srivastava K., Ahmed K. J., Fedds Repotoricim 105 (1994b) 185-189.

[13] Li M. H., Arch Environ Contam Toxicol. 45 (2003) 168-176.

[14] Liu Y. J., Ding H., Wseas Trans Environ Dev. 4 (2008) 24-32.

[15] Middleton J. T., Crafts A. S., Brewer R. F., Taylor 0. C., California agriculture (1956) 9-12.

[16] Naveed N. H., Aima Iram Batool A. I., Rehman U. F. Hameed U., African J. Environ. Sci. Tech. 4(11) (2010) 770-774.

[17] Rai A., K. Kulshreshtha, P. K. Srivastava, Mohanty C. S., Environmentalist 30 (2009) 18-23.

[18] Rai R., Rajput M., Agrawal M., Agrawal S. B., J Scientific Research 55 (2011) 77-102.

[19] Saquib M., Ahmad A., Ansari K., Ecoprint 17 (2010) 35-41.

[20] Seyyed M. S., Koochak H., International Conference on Environmental, Biomedical and Biotechnology, IPCBEE 16 (2011) 98-101.

[21] Singh S. K., Rao D. N., Proc. Symp on Air Pollution Control. 83 (1983) 218-224.

[22] Tiwari S., M. Agrawal, F. M., Marshall Environ Monitor Assess 119 (2006) 15-30.

[23] Tiwary S., Syed K., Sikka J., Joshi O. P., J. Env. Res. Dev. 2(3) (2008) 406-412.

[24] Tiwari S., Tiwari M., J. Environ. Res. Develop. 1(2) (2006) 129-135.

[25] Trivedi S., Agrawal M., Rajput M., Indian J Air Poll Cont 3 (2003) 44-53.

[26] Warren J. L., N.C. State University Sch. For resource Tech. Rep. 50 Raleigh. N.C. (1973). 